\title{
ON THE SCHUR, POSITIVE SCHUR AND WEAK DUNFORD-PETTIS PROPERTIES IN FRÉCHET LATTICES
}

\author{
Geraldo Botelho* and José Lucas P. Luiz ${ }^{\dagger}$ \\ Universidade Federal de Uberlândia, Faculdade de Matemática \\ 38.400-902 - Uberlândia - Brazil; botelho@ufu.br \\ IMECC-UNICAMP, Departamento de Matemática \\ 13.083-859 - Campinas - Brazil; lucasvt09@hotmail.com
}

\begin{abstract}
We prove some general results on sequential convergence in Fréchet lattices that yield, as particular instances, the following results regarding a closed ideal $I$ of a Banach lattice $E$ : (i) If two of the lattices $E, I$ and $E / I$ have the positive Schur property (the Schur property, respectively) then the third lattice has the positive Schur property (the Schur property, respectively) as well; (ii) If $I$ and $E / I$ have the dual positive Schur property, then $E$ also has this property; (iii) If $I$ has the weak Dunford-Pettis property and $E / I$ has the positive Schur property, then $E$ has the weak Dunford-Pettis property. Examples and applications are provided.
\end{abstract}

\section{Introduction}

In the realm of Banach spaces, the Schur property (weakly null sequences are norm null) is a 3 -space property in the weak sense that a Banach space $E$ has the Schur property whenever a closed subspace $F$ of $E$ and the quotient space $E / F$ have the Schur property (see, e.g., [8]). But it is not a 3-space property in the strong sense that, given a closed subspace $F$ of the Banach space $E$, if two of the spaces $E, F$ and $E / F$ have the Schur property, then the third one also has this property. To see that, just remember that $c_{0}$ is a quotient of $\ell_{1}$.

In the setting of Banach lattices, for the quotient $E / F$ of a Banach lattice $E$ over a closed subspace $F$ to be a Banach lattice, $F$ should be an ideal of $E$ (see, e.g., [3]). So the natural transposition of the concept of 3-space property (in the strong sense) to the realm of Banach lattices reads as follows.

Definition 1.1. A property $\mathcal{P}$ of Banach lattices is a 3-lattice property if the following holds: given a closed ideal $I$ of the Banach lattice $E$, if two of the lattices $E, I$ and $E / I$ have $\mathcal{P}$, then the third one has $\mathcal{P}$ as well.

A Banach lattice has the positive Schur property if weakly null sequences formed by positive vectors are norm null. A lot of research has been done on this property, for some recent contributions see, e.g., [6, 7, 13, 18, 24, 25, 26, 28]. Among other results, in this paper we prove that, contrary to the case of the Schur property for Banach spaces, the Schur and the positive Schur properties are 3-lattice properties. These results will appear as applications of general results on the Schur and the positive Schur properties in Fréchet lattices.

https://doi.org/10.5186/aasfm.2021.4642

2020 Mathematics Subject Classification: Primary 46A40, 46B42, 46A04.

Key words: Banach lattices, Fréchet lattices, Schur and positive Schur properties, dual positive Schur property, weak Dunford-Pettis property.

*Supported by CNPq Grant 304262/2018-8 and Fapemig Grant PPM-00450-17.

†Supported by a CNPq scholarship. 
Definition 1.2. Given linear topologies $\tau_{1}$ and $\tau_{2}$ in a Riesz space $E$, we say that:

- $E$ has the $\left(\tau_{1}, \tau_{2}\right)$-Schur property (in short, $\left(\tau_{1}, \tau_{2}\right)$-SP) if $\tau_{1}$-null sequences in $E$ are $\tau_{2}$-null.

- $E$ has the $\left(\tau_{1}, \tau_{2}\right)$-positive Schur property (in short, $\left(\tau_{1}, \tau_{2}\right)$-PSP) if $\tau_{1}$-null sequences in $E$ formed by positive elements are $\tau_{2}$-null.

Of course, the Schur property is the (weak topology, norm topology)-SP and the positive Schur property is the (weak topology, norm topology)-PSP in a Banach lattice. The first part of the definition above is similar to the approach of Castillo and Simões to the Schur property (see [9, Definition 4, Definition 5, Remark 5]).

After proving some general results on the $\left(\tau_{1}, \tau_{2}\right)$-SP and the $\left(\tau_{1}, \tau_{2}\right)$-PSP (see Theorems 2.1 and 2.3), we conclude in Corollary 2.4 that the Schur and the positive Schur properties are 3-lattices properties in the context of $\sigma$-Dedekind complete Fréchet lattices. Applying these results for Banach lattices we get, in Theorem 2.5, that the Schur and the positive Schur properties are 3-lattice properties, and in Proposition 2.12 that a Banach lattice $E$ has the dual positive Schur property whenever a closed ideal $I$ and the quotient lattice $E / I$ have this property. A few more applications to Banach lattices and an illustrative example are also provided. In a short final section we show that the methods we use for Fréchet lattices can also be helpful in the study of the weak Dunford-Pettis property in Banach lattices (cf. Theorem 3.2 and Corollary 3.3).

For the basic theory of Banach lattices, Riesz spaces and linear topologies on Riesz spaces we refer to $[1,2,3,17]$.

\section{The Schur and the positive Schur properties}

According to [2], a Fréchet lattice is a Riesz space endowed with a locally convexsolid (hence linear), metrizable and complete topology. We just recall that a locally convex topology on a Riesz space $E$ is locally convex-solid if it is generated by a family of Riesz seminorms, or, equivalently, if the origin has a basis of neighborhoods formed by convex and solid sets (see [3, Section 3.3]).

If $F$ is a Riesz subspace of the Risez space $E$ endowed with the linear topologies $\tau_{1}$ and $\tau_{2}$, we consider in $F$ the corresponding relative topologies, still denoted by $\tau_{1}$ and $\tau_{2}$. So, if $E$ has the $\left(\tau_{1}, \tau_{2}\right)$-SP $\left(\left(\tau_{1}, \tau_{2}\right)\right.$-PSP, respectively), then $F$ has the $\left(\tau_{1}, \tau_{2}\right)$-SP $\left(\left(\tau_{1}, \tau_{2}\right)\right.$-PSP, respectively $)$ as well.

Given a subspace $M$ of a topological vector space $(X, \tau)$, by $\dot{\tau}$ we denote the quotient topology on $X / M$, which is the finest topology on $X / M$ making the quotient operator $\pi: X \longrightarrow X / M$ continuous. It is well known that $\dot{\tau}$ is a linear topology on $X / M$ (see, e.g., [22, I.2.2]).

Given an ideal $I$ of the Riesz space $E$, the order in $E / I$ is given by: $\dot{x} \leq \dot{y}$ if there are $x_{1} \in \dot{x}$ and $y_{1} \in \dot{y}$ such that $x_{1} \leq y_{1}$ (see [3, pp. 99, 100] and [2, p. 16]).

The symbol $x_{n} \stackrel{\tau}{\longrightarrow} x$ means that the sequence $\left(x_{n}\right)_{n}$ converges to $x$ with respect to the topology $\tau$.

Theorem 2.1. Let $\tau_{1}$ and $\tau_{2}$ be linear topologies on the Riesz space $E$ such that $\left(E, \tau_{2}\right)$ is a Fréchet lattice and $E$ has the $\left(\tau_{2}, \tau_{1}\right)$-SP. Let $I$ be a $\tau_{2}$-closed ideal of $E$ such that $I$ has the $\left(\tau_{1}, \tau_{2}\right)-P S P\left(\left(\tau_{1}, \tau_{2}\right)-S P\right.$, respectively) and $E / I$ has the $\left(\dot{\tau}_{1}, \dot{\tau}_{2}\right)$-PSP $\left(\left(\dot{\tau}_{1}, \dot{\tau}_{2}\right)-S P\right.$, respectively). Then $E$ has the $\left(\tau_{1}, \tau_{2}\right)-P S P\left(\left(\tau_{1}, \tau_{2}\right)-S P\right.$, respectively). 
Proof. Let us prove first the case of the $\left(\tau_{1}, \tau_{2}\right)$-PSP. Let $\left(x_{n}\right)_{n} \subseteq E$ be a sequence such that $x_{n} \geq 0$ for every $n$ and $x_{n} \stackrel{\tau_{1}}{\longrightarrow} 0$. Of course, an arbitrary subsequence of $\left(x_{n}\right)_{n}$, still denoted by $\left(x_{n}\right)_{n}$, is positive and $\tau_{1}$-null. The continuity of the quotient operator $\pi:\left(E, \tau_{1}\right) \longrightarrow\left(E / I, \dot{\tau}_{1}\right)$ gives

$$
\dot{x}_{n}:=\pi\left(x_{n}\right) \stackrel{\dot{\tau}_{1}}{\longrightarrow} 0 \text { in } E / I,
$$

and the fact that $\pi$ is a Riesz homomorphism [3, Theorem 2.22] gives $\dot{x}_{n} \geq 0$ for every $n$. Calling on the $\left(\dot{\tau}_{1}, \dot{\tau}_{2}\right)$-PSP of $E / I$, we get $\dot{x}_{n} \stackrel{\dot{\tau}_{2}}{\longrightarrow} 0$ in $E / I$. Let $d$ be an invariant metric that generates the $\tau_{2}$ topology (see [1, Theorem 5.10]). Since $\dot{x}_{n} \stackrel{\dot{\tau}_{2}}{\longrightarrow} 0$ if and only if $\inf \left\{d\left(x_{n}, y\right): y \in I\right\} \longrightarrow 0$, there exists a sequence $\left(y_{n}\right)_{n}$ in $I$ and a further subsequence of $\left(x_{n}\right)_{n}$, still denoted by $\left(x_{n}\right)_{n}$, such that $d\left(x_{n}, y_{n}\right) \longrightarrow 0$, that is, $x_{n}-y_{n} \stackrel{\tau_{2}}{\longrightarrow} 0$ in $E$. Since $\tau_{2}$ is a locally convex-solid topology on $E$, and

$$
\left|x_{n}-\right| y_{n}||=|| x_{n}|-| y_{n}|| \leq\left|x_{n}-y_{n}\right|
$$

we get

$$
x_{n}-\left|y_{n}\right| \stackrel{\tau_{2}}{\longrightarrow} 0 \text { in } E .
$$

The $\left(\tau_{2}, \tau_{1}\right)$-SP of $E$ gives $x_{n}-\left|y_{n}\right| \stackrel{\tau_{1}}{\longrightarrow} 0$ in $E$, and since $x_{n} \stackrel{\tau_{1}}{\longrightarrow} 0$ we have $\left|y_{n}\right| \stackrel{\tau_{1}}{\longrightarrow} 0$ in $I$. Since $I$ has the $\left(\tau_{1}, \tau_{2}\right)$-PSP and each $\left|y_{n}\right| \in I$ is positive, it follows that $\left|y_{n}\right| \stackrel{\tau_{2}}{\longrightarrow} 0$ in $I$, hence $\left|y_{n}\right| \stackrel{\tau_{2}}{\longrightarrow} 0$ in $E$. Now the linearity of $\tau_{2}$ gives

$$
x_{n}=\left(x_{n}-\left|y_{n}\right|\right)+\left|y_{n}\right| \stackrel{\tau_{2}}{\longrightarrow} 0 \text { in } E .
$$

We have proved that every subsequence of $\left(x_{n}\right)_{n}$ admits a further subsequence that is $\tau_{2}$-null. This is enough to conclude that $\left(x_{n}\right)_{n}$ is itself $\tau_{2}$-null, proving that $E$ has the $\left(\tau_{1}, \tau_{2}\right)$-PSP.

The proof of the case of the $\left(\tau_{1}, \tau_{2}\right)$-SP follows the same steps, actually it is a bit easier because there is no need to pass to the absolute value of $y_{n}$.

In the particular case for the Schur property where $\tau_{1}$ is the weak topology on $E$ with respect to the linear topology $\left(E, \tau_{2}\right)$, we have $\tau_{1} \subseteq \tau_{2}$, hence $E$ has the $\left(\tau_{2}, \tau_{1}\right)$ SP. This means that, for this particular choice of $\tau_{1}$, the part about the Schur property of the theorem above collapses to [9, Proposition 6] (see also [8, Theorem 6.1.a]) due to Castillo and Simões, whose proof was our first inspiration.

To proceed we need the following lemma. Given a Riesz space $E$ endowed with a linear topology $\tau$, by $w_{\tau}$ we denote the weak topology on $E$ with respect to the topology $\tau$. It is well known that $w_{\tau}$ is a linear topology on $E$ (see, [20, 22]).

Lemma 2.2. If $(E, \tau)$ is a locally convex Hausdorff Riesz space with the $\left(w_{\tau}, \tau\right)$ $P S P$, then $(E, \tau)$ does not contain a lattice copy of $\ell_{\infty}$, meaning that there is no Riesz homomorphism from $\ell_{\infty}$ to $E$ that is a homeomorphism onto its range.

Proof. Suppose that there exists a Riesz homomorphism $T: \ell_{\infty} \longrightarrow E$ that is a homeomorphism onto $T\left(\ell_{\infty}\right)$. As $\tau$ is locally convex, by [20, Theorem 8.12.2] we know that the weak topology on $T\left(\ell_{\infty}\right)$ with respect to $\tau$ is the the weak topology $w_{\tau}$ on $E$ restricted to $T\left(\ell_{\infty}\right)$, which we still denote by $w_{\tau}$. Combining this with the $\left(w_{\tau}, \tau\right)$-PSP of $E$, it follows that $T\left(\ell_{\infty}\right)$ has the $\left(w_{\tau}, \tau\right)$-PSP as well. Since $T$ and $T^{-1}: T\left(\ell_{\infty}\right) \longrightarrow \ell_{\infty}$ are positive operators (obvious) and weak-weak continuous [20, Theorem 8.11.3(c)], we have that $\ell_{\infty}$ has the PSP. This contradiction completes the proof (to see that $\ell_{\infty}$ fails the PSP, note, e.g., that $c_{0}$ is a Banach sublattice of $\ell_{\infty}$ $\left[17\right.$, p. 12 , Example iii)] and the canonical unit vectors in $c_{0}$ form a non-norm null weakly null sequence of positive vectors in $c_{0}$ ). 
Remember that if $(E, \tau)$ is a Riesz space with a linear topology, then the weak topology $w_{\dot{\tau}}$ on $E / I$ with respect to the space $(E / I, \dot{\tau})$ coincides with the quotient topology $\dot{w}_{\tau}$ with respect to the space $\left(E, w_{\tau}\right)$ (see [20, Theorem 8.12.3(a)]).

Remember also that a Riesz space $E$ has the projection property if every band in $E$ is a projection band; and a locally solid Riesz space $(E, \tau)$ has the Lebesgue property if $x_{\alpha} \stackrel{\tau}{\longrightarrow} 0$ whenever $x_{\alpha} \downarrow 0$ in $E$ (here $\left(x_{\alpha}\right)_{\alpha}$ is a net in $E$ ). More details can be found in [2].

Theorem 2.3. Let $\tau$ be a locally convex-solid Hausdorff complete topology on the Dedekind $\sigma$-complete Riesz space $E$. If $E$ has the $\left(w_{\tau}, \tau\right)-P S P\left(\left(w_{\tau}, \tau\right)-S P\right.$, respectively), then, regardless of the $\tau$-closed ideal $I$ of $E$, the quotient space $E / I$ has the $\left(w_{\dot{\tau}}, \dot{\tau}\right)-P S P\left(\left(w_{\dot{\tau}}, \dot{\tau}\right)-S P\right.$, respectively $)$.

Proof. Assume that $E$ has the $\left(w_{\tau}, \tau\right)$-PSP. As $\tau$ is locally convex, by Lemma 2.2 we know that $(E, \tau)$ does not contain a lattice copy of $\ell_{\infty}$. Theorem 3.29 in [2] tells us that $(E, \tau)$ has the Lebesgue property, thus $E$ is Dedekind complete by [2, Theorem 3.24]. We conclude that $E$ has the projection property by [3, Theorem 1.42].

Let $I$ be a $\tau$-closed ideal of $E$. Since $(E, \tau)$ is locally solid with the Lebesgue property, calling [2, Theorem 3.7] we have that $I$ is a band, hence a projection band, which means that $E=I \oplus I^{d}$, where $I^{d}=\{x \in E: x \perp y$ for every $y \in I\}$. Using again that the weak topology on $I^{d}$ with respect to the $\tau$ is the restriction of the weak topology $w_{\tau}$ on $E$, the $\left(w_{\tau}, \tau\right)$-PSP of $E$ implies that $I^{d}$ has the $\left(w_{\tau}, \tau\right)$-PSP too. It follows from $[2$, p. 115$]$ that $(E / I, \dot{\tau})$ is lattice isomorphic to $\left(I^{d}, \tau\right)$, so $E / I$ has the $\left(w_{\dot{\tau}}, \dot{\tau}\right)$-PSP.

The case of the $\left(w_{\tau}, \tau\right)$-SP is similar. First observe that the $\left(w_{\tau}, \tau\right)$-SP is inherited by Riesz subspaces and is preserved by isomorphisms between locally convex spaces. Next follow the steps above and, as $(E / I, \dot{\tau})$ is lattice isomorphic to $\left(I^{d}, \tau\right)$ and this latter space has the $\left(w_{\tau}, \tau\right)$-SP, then $E / I$ has the $\left(w_{\dot{\tau}}, \dot{\tau}\right)$-SP.

As to the assumptions of the theorem above, recall that, as in the case of Banach spaces, a linear subspace of a locally convex space is closed if and only if it is weakly closed [20, Theorem 8.8.1].

Combining Theorems 2.1 and 2.3 we get the following:

Corollary 2.4. The (weak, strong)-positive Schur property and the (weak, strong)-Schur property are 3-lattice properties in the context of $\sigma$-Dedekind complete Fréchet lattices in the following sense: if $I$ is a closed ideal of a $\sigma$-Dedekind complete Fréchet lattice $(E, \tau)$ such that two out of the three following conditions hold:

(i) $E$ has the $\left(w_{\tau}, \tau\right)-P S P\left(\left(w_{\tau}, \tau\right)-S P\right.$, respectively),

(ii) I has the $\left(w_{\tau}, \tau\right)-P S P\left(\left(w_{\tau}, \tau\right)-S P\right.$, respectively),

(iii) $E / I$ has the $\left(\dot{w}_{\tau}, \dot{\tau}\right)-P S P\left(\left(\dot{w}_{\tau}, \dot{\tau}\right)-S P\right.$, respectively);

then the third condition holds too.

The notion of 3-lattice properties in Banach lattices was defined in the Introduction.

Theorem 2.5. The Schur and the positive Schur properties are 3-lattice properties.

Proof. Since $c_{0}$ fails the positive Schur property, Banach lattices with the Schur/ positive Schur property do not contain a copy of $c_{0}$, hence they are KB-spaces [3, Theorem 4.60]. But KB-spaces have order continuous norms [3, p. 232] and Banach lattices with order continuous norms are Dedekind complete [3, Corollary 4.10]. So, 
Banach lattices with the Schur/positive Schur property are Dedekind complete. Of course, in Banach lattices the Schur property is the (weak, norm)-SP and the positive Schur property is the (weak,norm)-PSP, so the result follows from Corollary 2.4.

Next we give applications and examples regarding the results obtained thus far.

Proposition 2.6. Let $E$ and $F$ be Banach lattices and $T: E \longrightarrow F$ be a Riesz homomorphism with closed range. If $\operatorname{ker}(T)$ and $F$ have the positive Schur property (Schur property, respectively), then $E$ has the positive Schur property (Schur property, respectively).

Proof. Let us prove the case of the positive Schur property. As a Banach sublattice of $F, T(E)$ has the positive Schur property. We believe it is well known that $E / \operatorname{ker}(T)$ is lattice isomorphic to $T(E)$, but since we have found no reference to quote, we give a short reasoning. Since $T$ is a Riesz homomorphism, $\operatorname{ker}(T)$ is an ideal of $E$ [3, p. 94]), and the continuity of $T$ (Riesz homomorphisms are positive hence continuous) guarantees that $\operatorname{ker}(T)$ is closed. So $E / \operatorname{ker}(T)$ is a Banach lattice. The operator

$$
S: E / \operatorname{ker}(T) \longrightarrow T(E), \quad S(\dot{x})=T(x)
$$

is an isomorphism between Banach spaces such that $S \circ \pi=T$, where $\pi: E \longrightarrow$ $E / \operatorname{ker}(T)$ is the quotient operator [16, Theorem 1.7.14]. From the fact that $T$ and $\pi$ are Riesz homomorphisms it follows easily that $S$ is a Riesz homomorphism as well, so $E / \operatorname{ker}(T)$ is lattice isomorphic to $T(E)$. Therefore $E / \operatorname{ker}(T)$ has the positive Schur property and Theorem 2.5 gives that $E$ has the positive Schur property. The case of the Schur property is identical.

On the one hand, higher order duals $E^{* *}, E^{* * *}, \ldots$ of infinite dimensional Banach spaces $E$ never have the Schur property (this follows from [19, Corollary 11]). On the other hand, the bidual of an infinite dimensional Banach lattice with the positive Schur property may have the positive Schur property. For example, every AL-space has the positive Schur property [21, Examples 1.3] and the bidual of an AL-space is an AL-space as well [3, Theorem 4.23]. We believe that, contrary to the case of the Schur property in Banach spaces, it is not easy to give concrete examples of Banach lattices $E$ with the positive Schur property such that $E^{* *}$ fails this property. In this case, is $E$ a closed ideal of $E^{* *}$ ? If yes, our results imply that $E^{* *} / E$ fails the positive Schur property. Our next aim is to give such an example.

Proposition 2.7. Let $E$ be a Banach lattice with the positive Schur property such that $E^{*}$ contains a lattice copy of $\ell_{1}$. Then $E$ is a closed ideal of $E^{* *}$ and $E^{* *}$ and $E^{* *} / E$ fail the positive Schur property.

Proof. We have already noticed that Banach lattices with the positive Schur property do not contain a lattice copy of $c_{0}$, so $E$ does not contain a lattice copy of $c_{0}$, therefore $E$ is a KB-space (we have already used this fact). By [3, Theorem 4.60] we know that $E$ is a band, in particular a closed ideal, of $E^{* *}$. So we can consider the quotient lattice $E^{* *} / E$. Since $E^{*}$ contains a lattice copy of $\ell_{1}$, from a result due to Wnuk [25, p. 22] it follows that $E^{* *}$ fails the positive Schur property. Corollary 2.5 gives that $E^{* *} / E$ lacks the positive Schur property.

Example 2.8. For each $n \in \mathbf{N}$ let $\ell_{n}^{\infty}$ denote the Banach lattice $\mathbf{R}^{n}$ endowed with the maximum norm and coordinatewise order. Now let $E$ denote the $\ell_{1}$-sum of 
the sequence $\left(\ell_{n}^{\infty}\right)_{n}$, that is

$E:=\left(\bigoplus_{n \in \mathbf{N}} \ell_{n}^{\infty}\right)_{1}=\left\{x=\left(x_{n}\right)_{n}: x_{n} \in \ell_{n}^{\infty}\right.$ for every $n \in \mathbf{N}$ and $\left.\|x\|:=\sum_{n=1}^{\infty}\left\|x_{n}\right\|<\infty\right\}$,

which is a Banach lattice endowed with the coordinatewise order. As a finite dimensional Banach lattice, each $\ell_{n}^{\infty}$ has the positive Schur property, so $E$ has the positive Schur property by another result due to Wnuk [25, p. 17].

Let us see now that $E^{*}$ contains a lattice copy of $\ell_{1}$. To do so, first remember that $\left(\ell_{n}^{\infty}\right)^{*}$ is canonically lattice isometric to $\ell_{n}^{1}=\left(\mathbf{R}^{n},\|\cdot\|_{1}\right)$. So, by [3, Theorem 4.6] we know that $E^{*}$ is lattice isometric to

$$
\left(\bigoplus_{n \in \mathbf{N}} \ell_{n}^{1}\right)_{\infty}:=\left\{x=\left(x_{n}\right)_{n}: x_{n} \in \ell_{n}^{1} \text { for every } n \in \mathbf{N} \text { and }\|x\|:=\sup _{n}\left\|x_{n}\right\|<\infty\right\} \text {, }
$$

with the coordinatewise order, via the usual duality relation

$$
\left(\varphi_{j}\right)_{j} \longmapsto\left(\varphi_{j}\right)_{j}\left(\left(x_{j}\right)_{j}\right)=\sum_{j=1}^{\infty} \varphi_{j}\left(x_{j}\right) .
$$

It is well know that the map

$$
\left(x_{j}\right)_{j} \in \ell_{1} \longmapsto\left(\left(x_{1}\right),\left(x_{1}, x_{2}\right),\left(x_{1}, x_{2}, x_{3}\right), \ldots\right) \in\left(\bigoplus_{n \in \mathbf{N}} \ell_{n}^{1}\right)_{\infty},
$$

is a linear isometric embedding and a Riesz homomorphism. So, $E^{*}$ contains a lattice copy of $\ell_{1}$, then Proposition 2.7 yields that $E$ is a closed ideal of $E^{* *}$ and that the Banach lattices

$$
\left(\bigoplus_{n \in \mathbf{N}} \ell_{n}^{\infty}\right)_{1}^{* *} \text { and }\left(\bigoplus_{n \in \mathbf{N}} \ell_{n}^{\infty}\right)_{1}^{* *} /\left(\bigoplus_{n \in \mathbf{N}} \ell_{n}^{\infty}\right)_{1}
$$

lack the positive Schur property.

It is well known that $\ell_{1}$ is projectively universal for the class of separable Banach spaces in the sense that every separable Banach space is isometric to a quotient of $\ell_{1}$. The fact that the Schur property is preserved under isomorphisms and Theorem 2.5 give immediately the following.

Corollary 2.9. Let $E$ be a separable Banach space failing the Schur property. Then any closed subspace $M$ of $\ell_{1}$ such that $E$ is isomorphic to $\ell_{1} / M$ fails to be an ideal.

Although $\ell_{1}$ is a Banach lattice, it is not true that every separable Banach lattice is lattice isometric to a quotient of $\ell_{1}$ over a closed ideal. For example, $c_{0}$ is a separable Banach lattice that is not lattice isometric to a quotient of $\ell_{1}$ over a closed ideal (otherwise $c_{0}$ would be an AL-space because the quotient of an AL-space over a closed ideal is an AL-space [3, p. 205]). In [15], Leung, Li, Oikhberg and Tursi constructed a separable Banach lattice LLOT such that every separable Banach lattice is lattice isometric to a quotient of $L L O T$ over a closed ideal.

Example 2.10. Let us see that the Banach lattice LLOT lacks the positive Schur property. Of course this is the case if it contains a lattice copy of $c_{0}$. Suppose that $L L O T$ does not contain a lattice copy of $c_{0}$ and has the positive Schur property. In this case $L L O T$ would be $\sigma$-Dedekind complete and, since the positive Schur property is preserved by lattice isomorphisms of Banach lattices, from Theorem 2.3 it would follow that every separable Banach lattice has this property. 
The existence of separable Banach lattices failing the positive Schur property, $\ell_{2}$ for example, completes the reasoning.

Remark 2.11. In [11], Flores, Hernández, Spinu, Tradacete and Troitsky introduced and developed the notion of $p$-disjointly homogeneous Banach lattices, $1 \leq p \leq \infty$. In [11, Proposition 4.9] they proved that a Banach lattice is 1-disjointly homogeneous if and only if it has the positive Schur property. So, being a 1-disjointly homogeneous Banach lattice is a 3-lattice property.

We finish this section with an application of our results to the dual positive Schur property, introduced by Aqzzouz, Elbour and Wickstead [5] as follows: a Banach lattice $E$ has the dual positive Schur property if every weak*-null sequence formed by positive functionals in $E^{*}$ is norm null.

Let us see first that the dual positive Schur property is not a 3-lattice property. According to Wnuk [27, p. 768], $\ell_{\infty}$ and $\ell_{\infty} / c_{0}$ have the dual positive Schur property. But $c_{0}$ is a closed ideal of $\ell_{\infty}$ lacking the dual positive Schur property (the canonical unit vectors form a weak ${ }^{*}$-null non-norm null sequence in $\ell_{1}$ ). We have just mentioned that Wnuk [27] established that the dual positive Schur property passes to quotients over closed ideals. So, all that is left to be established is the following:

Proposition 2.12. If the closed ideal I of the Banach lattice $E$ and the quotient lattice $E / I$ have the dual positive Schur property, so has $E$.

Proof. For a Banach space $X$, let us call $\sigma\left(X^{*}, X\right)$ the weak*-topology on $X^{*}$ and $\|\cdot\|_{X^{*}}$ the norm topology on $X^{*}$. So,

- $E$ has the dual positive Schur property if and only if $E^{*}$ has the $\left(\sigma\left(E^{*}, E\right), \|\right.$. $\left.\|_{E^{*}}\right)$-PSP;

- $I$ has the dual positive Schur property if and only if $I^{*}$ has the $\left(\sigma\left(I^{*}, I\right),\|\cdot\|_{I^{*}}\right)$ PSP;

- $E / I$ has the dual positive Schur property if and only if $(E / I)^{*}$ has the $\left(\sigma\left((E / I)^{*}, E / I\right),\|\cdot\|_{(E / I)^{*}}\right)$-PSP.

Since the annihilator $I^{\perp}$ of $I$ is a closed ideal of $E^{*}[23$, Corollary 1 of Proposition II.5.5], combining [10, Theorems V.2.2 and V.2.3] and [23, Corollary 1 of Proposition II.5.5] we get

- $I^{*}$ has the $\left(\sigma\left(I^{*}, I\right),\|\cdot\|_{I^{*}}\right)$-PSP if and only if $E^{*} / I^{\perp}$ has the $\left(\left[\sigma\left(E^{*}, E\right)\right]\right.$, $\left.\|\cdot\|_{E^{*}}\right)$-PSP, where $\left[\sigma\left(E^{*}, E\right)\right]$ is the quotient topology associated to the quotient operator $\left(E^{*}, \sigma\left(E^{*}, E\right)\right) \longrightarrow E^{*} / I^{\perp}$, and

- $(E / I)^{*}$ has the $\left(\sigma\left((E / I)^{*}, E / I\right),\|\cdot\|_{(E / I)^{*}}\right)$-PSP if and only if $I^{\perp}$ has the $\left(\sigma\left(E^{*}, E\right),\|\cdot\|_{E^{*}}\right)$-PSP.

The assumptions give that $I^{\perp}$ has the $\left(\sigma\left(E^{*}, E\right),\|\cdot\|_{E^{*}}\right)$-PSP and that the quotient lattice $E^{*} / I^{\perp}$ has the $\left(\left[\sigma\left(E^{*}, E\right)\right],\|\cdot\|_{E}\right)$-PSP, therefore Theorem 2.1 yields that $E^{*}$ has the $\left(\sigma\left(E^{*}, E\right),\|\cdot\|_{E^{*}}\right)$-PSP, that is, $E$ has the dual positive Schur property.

\section{The weak Dunford-Pettis property}

The weak Dunford-Pettis property was introduced by Leung [14] as follows: a Banach lattice $E$ has the weak Dunford-Pettis property if every weakly compact operator on $E$ sends weakly null sequences formed by pairwise disjoint vectors to norm null sequences. In [8], the authors mention that it was then unknown if this property is a 3-lattice property. As far as we know, this problem remains open, and 
the purpose of this short final section is to show that the methods we are applying can be used to give a contribution in this direction.

Following the approach of Gabriyelyan [12], we give the following definition in the context of Fréchet lattices.

Definition 3.1. A Fréchet lattice $E$ has the sequential weak Dunford-Pettis property if $\varphi_{n}\left(x_{n}\right) \longrightarrow 0$ whenever $\left(x_{n}\right)_{n}$ is a weakly null sequence in $E$ formed by positive vectors and $\left(\varphi_{n}\right)_{n}$ is a weakly null sequence in the strong dual $E_{\beta}^{*}$ of $E$.

The next result was inspired by [8, Proposition 6.6.c] and its proof repeats some of the steps of the proof of Theorem 2.1.

Theorem 3.2. Let $I$ be a closed ideal of the Fréchet lattice $(E, \tau)$. If I has the sequential weak Dunford-Pettis property and $E / I$ has the $\left(\dot{w}_{\tau}, \dot{\tau}\right)-P S P$, then $E$ has the sequential weak Dunford-Pettis property.

Proof. Given a weakly null sequence $\left(x_{n}\right)_{n}$ of positive vectors of $E$ and a weakly null sequence $\left(\varphi_{n}\right)_{n}$ in $E_{\beta}^{*}$, let us denote an arbitrary subsequence of $\left(\varphi_{n}\left(x_{n}\right)\right)_{n}$ still by $\left(\varphi_{n}\left(x_{n}\right)\right)_{n}$. Of course, the corresponding subsequences of $\left(x_{n}\right)_{n}$ and of $\left(\varphi_{n}\right)_{n}$ satisfy the same properties of the original sequences. Considering the quotient operator $\pi:(E, \tau) \longrightarrow(E / I, \dot{\tau})$, we have that $\left(\dot{x}_{n}\right)_{n}=\left(\pi\left(x_{n}\right)\right)_{n}$ is a sequence of positive vectors in $E / I$ and $\dot{x}_{n} \stackrel{\dot{w}_{\tau}}{\longrightarrow} 0$ in $E / I$. The $\left(\dot{w}_{\tau}, \dot{\tau}\right)$-PSP of $E / I$ gives $\dot{x}_{n} \stackrel{\dot{\tau}}{\longrightarrow} 0$ in $E / I$ and reasoning similarly to the proof of Theorem 2.1 we can find a further subsequence of $\left(x_{n}\right)_{n}$, still denoted by $\left(x_{n}\right)_{n}$, and a sequence $\left(y_{n}\right)_{n}$ of positive vectors of $I$ such that, up to an adjustment of the indexes if necessary, $x_{n}-y_{n} \stackrel{\tau}{\longrightarrow} 0$. Hence, $x_{n}-y_{n} \stackrel{w_{\tau}}{\longrightarrow} 0$ in $E$ and from the linearity of the weak topology we get

$$
y_{n}=x_{n}-\left(x_{n}-y_{n}\right) \stackrel{w_{\tau}}{\longrightarrow} 0
$$

in $E$, thus in $I$. Since $\left(\varphi_{n}\right)_{n}$ is weakly null in $E_{\beta}^{*}$, denoting by $\varphi_{\left.n\right|_{I}}$ the restriction of each $\varphi_{n}$ to $I$ and applying [20, Theorem 8.11.3(d) and (c)] to the inclusion operator $(I, \tau) \longrightarrow(E, \tau)$ and its adjoint $E_{\beta}^{*} \longrightarrow I_{\beta}^{*}$, we conclude that $\left(\varphi_{\left.n\right|_{I}}\right)_{n}$ is weakly null in the strong dual $I_{\beta}^{*}$ of $I$. The sequential weak Dunford-Pettis property of $I$ gives $\varphi_{\left.n\right|_{I}}\left(y_{n}\right) \longrightarrow 0$, that is, $\varphi_{n}\left(y_{n}\right) \longrightarrow 0$.

On the other hand, $\left(\varphi_{n}\right)_{n}$, along with all its subsequences, is pointwise bounded because it is weakly null in $E_{\beta}^{*}$, therefore it is equicontinuous by the Banach-Steinhaus Theorem [20, Theorem 11.9.1] (remember that $E$ is a Fréchet space). So, given $\varepsilon>0$ there is a 0 -neighborhood $U$ in $E$ such that $\left|\varphi_{n}(x)\right|<\varepsilon$ for every $x \in U$. Now the convergence $x_{n}-y_{n} \stackrel{\tau}{\longrightarrow} 0$ implies that $\varphi_{n}\left(x_{n}-y_{n}\right) \longrightarrow 0$, therefore

$$
\varphi_{n}\left(x_{n}\right)=\varphi_{n}\left(x_{n}-y_{n}\right)+\varphi_{n}\left(y_{n}\right) \longrightarrow 0 .
$$

We have proved that every subsequence of $\left(\varphi_{n}\left(x_{n}\right)\right)_{n}$ admits a further null subsequence. This is enough to conclude that $\varphi_{n}\left(x_{n}\right) \longrightarrow 0$, and then $E$ has the sequential weak Dunford-Pettis property.

Corollary 3.3. Let $I$ be a closed ideal of the Banach lattice $E$. If $I$ has the weak Dunford-Pettis property and E/I has the positive Schur property, then $E$ has the weak Dunford-Pettis property.

Proof. Since the strong topology $\beta\left(E^{*}, E\right)$ on the dual $E^{*}$ of a Banach space $E$ coincides with the norm topology [20, Example 8.8.9], the weak sequential DunfordPettis property coincides with the weak Dunford-Pettis property in a Banach lattice (see [4, Corollary 2.6]), so the result follows from Theorem 3.2. 
Acknowledgements. The authors are grateful to Khazhak V. Navoyan for her helpful suggestions and to the referee for her/his careful reading of the manuscript and the suggestions that improved the final presentation of the paper.

\section{References}

[1] Aliprantis, C., and K. Border: Infinite dimensional analysis: a hitchhiker's guide. Springer, Berlin-Heidelberg-New York, third ed., 2006.

[2] Aliprantis, C., and O. Burkinshaw: Locally solid Riesz spaces with applications to economics. Second edition. - Amer. Math. Soc., 2003.

[3] Aliprantis, C., and O. Burkinshaw: Positive operators. - Springer, Dordrecht, 2006.

[4] Aqzzouz, B., and A. Elbour: Some characterizations of almost Dunford-Pettis operators and applications. - Positivity 15:3, 2011, 369-380.

[5] Aqzzouz, B., A. Elbour, and A. W. Wickstead: Positive almost Dunford-Pettis operators and their duality. - Positivity 15:2, 2011, 185-197.

[6] Ardakani, H., S. M. Moshtaghioun, S. M. S. M. Mosadegh, and M. Salimi: The strong Gelfand-Phillips property in Banach lattices. - Banach J. Math. Anal. 10, 2016, 15-26.

[7] Baklouti, H., and M. Hajus: Schur operators and domination problem. - Positivity 21, 2017, $35-48$.

[8] Castillo, J. M. F., and M. GonzÁlez: Three-space problems in Banach space theory. Lecture Notes in Math. 1667, Springer, Berlim, 1997.

[9] Castillo, J. M. F., and M. A. Simões: On $p$-summable sequences in locally convex spaces. Extracta Math. 18, 2003, 209-222.

[10] Conway, J. B.: A course in functional analysis. Second Edition. - Springer, New York, 1990.

[11] Flores, J., F. L. Hernández, E. Spinu, P. Tradacete, and V. G. Troitsky: Disjointly homogeneous Banach lattices: duality and complementation. - J. Funct. Anal. 226, 2014, 58585885 .

[12] Gabriyelyan, S.: Locally convex spaces and Schur type properties. - Ann. Acad. Sci. Fenn. Math. 44:1, 2019, 363-378.

[13] Kaminska, A., and M. Mastylo: The Schur and (weak) Dunford-Pettis properties in Banach lattices. - J. Austral. Math. Soc. 73, 2002, 251-278.

[14] Leung, D. H.: On the weak Dunford-Pettis property. - Arch. Math. 52, 1989, 363-364.

[15] Leung, D. H., L. Li, T. Oikhberg, and M. A. Tursi: Separable universal Banach lattices. - Israel J. Math. 230, 2019, 141-152.

[16] Megginson, R. R.: An introduction to Banach space theory. - Grad. Texts in Math., Springer, 1998.

[17] Meyer-Nieberg, P.: Banach lattices. - Springer Verlag, Berlin, Heidelberg, New York, 1991.

[18] Moussa, M., and K. Bouras: About positive weak Dunford-Pettis operators on Banach lattices. - J. Math. Anal. Appl. 381, 2011, 891-896.

[19] MujicA, J.: Banach spaces not containing $\ell_{1}$. - Ark. Mat. 41:2, 2003, 363-374.

[20] Narici, L., and E. Beckenstein: Topological vector spaces. Second edition. - Chapman and Hall-CRC, Boca Raton-London-New York, 2010.

[21] RÄBigeR, F.: Lower and upper 2-estimates for order bounded sequences and Dunford-Pettis operators between certain classes of Banach lattices. - Lecture Notes in Math. 1470, 1991, $159-170$.

[22] Schaefer, H. H.: Topological vector spaces. Third edition. - Springer-Verlag, New YorkHeidelberg-Berlin, 1970. 
[23] Schaefer, H. H.: Banach lattices and positive operators. - Springer-Verlag, New YorkHeidelberg-Berlin, 1974.

[24] Tradacete, P.: Positive Schur properties in spaces of regular operators. - Positivity 19, 2015, $305-316$.

[25] Wnuk, W.: Banach lattices with properties of the Schur type: a survey. - Conf. Sem. Mat. Univ. Bari 249, 1993, 1-25.

[26] Wnuk, W.: The strong Schur property in Banach lattices. - Positivity 13, 2009, 435-441.

[27] Wnuk, W.: On the dual positive Schur property in Banach lattices. - Positivity 17, 2013, 759-773.

[28] Zeekoei, E. D., and J.H. Fourie: On p-convergent operators on Banach lattices. - Acta Math. Sin. (Engl. Ser.) 34:5, 2018, 873-890.

Received 21 April $2020 \bullet$ Accepted 11 September 2020 